\title{
Dysregulated genes and miRNAs in the apoptosis pathway in colorectal cancer patients
}

\author{
Martha L. Slattery ${ }^{1}$ (1) $\cdot$ Lila E. Mullany $^{1} \cdot$ Lori C. Sakoda $^{2} \cdot$ Roger K. Wolff $^{1} \cdot$ Wade S. Samowitz $^{3} \cdot$ Jennifer S. Herrick $^{1}$
}

Published online: 7 March 2018

(c) The Author(s) 2018. This article is an open access publication

\begin{abstract}
Apoptosis is genetically regulated and involves intrinsic and extrinsic pathways. We examined 133 genes within these pathways to identify whether they are expressed differently in colorectal carcinoma (CRC) and normal tissue $(\mathrm{N}=217)$ and if they are associated with similar differential miRNA expression. Gene expression data (RNA-Seq) and miRNA expression data (Agilent Human miRNA Microarray V19.0) were generated. We focused on dysregulated genes with a fold change (FC) of $>1.50$ or $<0.67$, that were significant after adjustment for multiple comparisons. miRNA:mRNA seed-region matches were determined. Twenty-three genes were significantly downregulated $(\mathrm{FC}<0.67)$ and 18 were significantly upregulated (FC $>1.50)$. Of these 41 genes, 11 were significantly associated with miRNA differential expression. BIRC5 had the greatest number of miRNA associations (14) and the most miRNAs with a seed-region match (10). Four of these matches, miR-145-5p, miR-150-5p, miR-195-5p, and miR-650, had a negative beta coefficient. CSF2RB was associated with ten total miRNAs (five with a seed-region match, and one miRNA, miR-92a-3p, with a negative beta coefficient). Of the three miRNAs associated with CTSS, miR-20b-5p, and miR-501-3p, had a seed-region match and a negative beta coefficient between miRNA:mRNA pairs. Several miRNAs that were associated with dysregulated gene expression, seed-region matches, and negative beta coefficients also were associated with CRC-specific survival. Our data suggest that miRNAs could influence several apoptosis-related genes. BIRC5, CTSS, and CSF2R all had seed-region matches with miRNAs that would favor apoptosis. Our study identifies several miRNA associated with apoptosis-related genes, that if validated, could be important therapeutic targets.
\end{abstract}

Keywords Apoptosis $\cdot$ Colorectal cancer $\cdot$ miRNA $\cdot$ mRNA $\cdot$ BIRC5 $\cdot$ CTSS

\section{Introduction}

Programmed cell death, or apoptosis, is genetically regulated [1]. Apoptosis is a homeostatic mechanism by which cell populations in tissues are maintained; it is a coordinated and

Electronic supplementary material The online version of this article (https://doi.org/10.1007/s10495-018-1451-1) contains supplementary material, which is available to authorized users.

Martha L. Slattery

marty.slattery@hsc.utah.edu

1 Department of Medicine, University of Utah, 383 Colorow, Salt Lake City, UT 84108, USA

2 Division of Research, Kaiser Permanente Northern California, Oakland, CA, USA

3 Department of Pathology, University of Utah, Salt Lake City, UT, USA energy-dependent process that involves activation of caspases (CASP), a group of cysteine proteases, followed by a cascade of events that ultimately end in cell death. Major caspases are classified into initiators of apoptosis (CASP 2, 8, 9, and 10), executioners of apoptosis (CASP 3, 6, and 7), and inflammatory caspases (CASP 1, 4, and 5). Activation of CASP8 triggers the execution phase of apoptosis; CASP 3 is thought to be the most important executioner caspase. The two main linked pathways in apoptosis are the extrinsic or death receptor pathway and the intrinsic or mitochondrial pathway. The extrinsic signaling pathway includes tumor necrosis factor (TNF) receptor gene superfamily. The intrinsic signaling pathway involves mechanisms whereby signals can have either a positive or negative affect on apoptosis. Negative signals from the lack of specific growth factors, hormones, or cytokines can result in loss of apoptotic suppression and hence the activation of apoptosis. These changes also influence the mitochondrial membrane, activating the mitochondrial pathway. Control and regulation 
of these apoptotic mitochondrial events involves members of the Bcl-2 protein family. Members of the Bcl-2 protein family include anti-apoptotic proteins such as $\mathrm{Bcl}-2, \mathrm{Bcl}-\mathrm{X}, \mathrm{Bcl}-$ $\mathrm{XL}, \mathrm{Bcl}-\mathrm{XS}, \mathrm{BclW}$ and $\mathrm{Bag}$ as well as pro-apoptotic proteins such as Bcl-10, Bax, Bak, Bid, Bad, Bim, Bik, and Blk. These proteins are central in determining if apoptosis occurs. The IAP (inhibitor of apoptosis) family of proteins, which contains the Baculoviral IAP Repeat Containing (BIRC) genes, is an important regulator of apoptosis in that these proteins regulate both the extrinsic and intrinsic pathways.

MicroRNAs (miRNAs), small noncoding RNAs that bind to the 3'UTR of the protein coding mRNAs and inhibit their translation, may also be transcriptional targets. MiRNAs have been shown to be involved in many cellular processes, one of which is apoptosis. Several miRNAs, including miR-124 [2], miR-195 [3], miR-148a [4], miR-365 [5], miR-125b [6], miR-129 [7], miR-143 [8], and miR-203 [9], have been linked to apoptosis through regulation of genes such as BCL2 and PUMA, a member of the Bcl-2 family, involved in pro-apoptosis $[1,4,6,8,9]$.

Most studies of miRNAs and apoptosis have targeted specific miRNAs along with specific proteins or genes. In this study, we comprehensively evaluated all apoptosis genes identified in the Kyoto Encyclopedia of Genes and Genomes (KEGG) pathway to identify which genes are dysregulated in colorectal cancer (CRC). We compare dysregulated apoptosis genes with differentially expressed miRNAs to better define how miRNAs influence apoptosis. We utilized seed matches between associated mRNAs and miRNAs to determine if the associations are more likely to be direct, in that the binding of the miRNA to the mRNA directly influences the gene expression, or if the association is an indirect biological function in which the association between the miRNA and the mRNA is more likely to be from a feedback loop. A seed match would increase the likelihood that an identified miRNA:mRNA interaction was more likely to have a direct biological effect on expression given a higher propensity for binding. We further evaluated the impact of these dysregulated genes and miRNAs on CRC-specific survival.

\section{Methods}

\section{Study participants}

This study incorporates data from 217 individuals who participated in one of two population-based case-control studies of incident colon and rectal cancer patients in Utah or were members of Kaiser Permanente Northern California (KPNC). Participants were between 30 and 79 years of age, and self-reported being either non-Hispanic white, Hispanic, black, or Asian (rectal cancer study) [10, 11]. SEER (Surveillance, Epidemiology, and End Results) registries were used to verify cases as a first primary adenocarcinoma of the colon or rectum within the study-specific dates (October 1991 to September 1994 for the colon study or between May 1997 and May 2001 for the rectal study) [12]. SEER Registries in Northern California and Utah also provided information on tumor site, date of diagnosis, date of death or lost to follow-up, months of survival after diagnosis until either death or end of follow-up, and cause of death. The study was approved by the Institutional Review Boards at the University of Utah and at KPNC.

\section{RNA}

Methods for RNA extraction, processing, and analysis have been described in detail $[13,14]$. RNA was extracted from formalin-fixed paraffin embedded tissue from the initial biopsy or surgery, prior to chemotherapy treatment. For both mRNA and miRNA analysis, RNA was extracted from paired samples that consisted of the carcinoma tissue and adjacent normal mucosa as previously described [15]. Of the 245 individuals with sufficient tissue for analysis, 217 passed quality control (QC) were used in these analyses [16]. A more detailed description of the methods can be found in our previous work [17]. Total gene counts were calculated using gene coordinates obtained from http://genome.ucsc. edu. Genes that were not expressed in our RNA-Seq data or for which the expression was missing for the majority of samples were excluded from further analysis [17]. The Agilent Human miRNA Microarray V19.0 was used to generate miRNA data. QC parameters established by Agilent were implemented as previously described. Our previous assessment of miRNA expression repeatability was extremely high $(r=0.98)$ [12]. Further comparison of expression and fold change (FC) between paired tissue for the Agilent microarray with qPCR expression results showed $100 \%$ agreement when considering directionality of findings (i.e. expression either upregulated or downregulated in carcinoma tissue compared to normal) with FC being almost identical [18]. MiRNA expression was normalized by a scaling factor that was the median of the 75th percentile of all samples divided by the 75th percentile for each individual sample [19].

\section{KEGG-identified apoptosis genes}

The KEGG pathway map program was used to identify a comprehensive list of genes within the apoptosis pathway (http://www.genome.jp/kegg-gin/show_pathway?hsa04210). We identified 138 genes (Supplemental Table S1) in this signaling pathway; 133 of these genes had sufficient expression in CRC tissue for statistical analysis. 


\section{Statistical methods}

We utilized a negative binomial mixed effects model in SAS, taking into account carcinoma/normal status as well as subject effect, to determine which genes had statistically significant difference in expression, either upregulated or downregulated, between paired carcinoma and normal mucosa. The log of the expression of all identified protein-coding genes in the negative binomial model $(n=17,461)$ was used to offset the overall exposure. The level of expression of each gene was calculated by dividing an individual's total gene expression by the total expression of all protein-coding genes per million transcripts (RPMPCG or reads per million protein-coding genes). We calculated each individual's FC between their carcinoma and normal mucosa expression. Our analysis with mRNAs and miRNAs focused on FCs of $>1.50$ or $<0.67$. The Benjamini and Hochberg [20] method was used to control the false discovery rate (FDR) using a value of $<0.05$; this served as an adjustment for multiple comparisons. We considered overall CRC differential expression as well as differential expression specific for microsatellite unstable (MSI) and microsatellite stable (MSS) tumors to help determine genes that may have unique tumor phenotype associations.

We fit a least squares linear regression model to the RPMPCG differential expression levels and miRNA differential expression levels to determine mRNA:miRNA associations. We used the bootstrap method by creating a distribution of $10,000 \mathrm{~F}$ statistics derived by resampling the residuals from the null hypothesis model of no association between gene expression and miRNA expression using the boot package in $\mathrm{R}$ to generate $\mathrm{p}$ values. Our linear models were adjusted for age and sex. Multiplicity adjustments for gene $\mathrm{mRNA} / \mathrm{miRNA}$ associations were made using the FDR by Benjamini and Hochberg [20].

Survival analyses were conducted using survival months calculated from diagnosis date to date of death or last follow-up, whichever occurred earlier. CRC-specific follow-up included deaths where the primary or secondary cause of death was listed as CRC. Individuals dying of other causes or who were lost to follow-up were censored at their time of death or date of last contact. We utilized the R package "survival" to calculate $p$ values based upon 10,000 permutations of the likelihood ratio test from the Cox proportional hazards model adjusted for age at diagnosis, gender, and AJCC tumor stage at diagnosis. Reported Hazard Ratios (HR) are based on the difference between the 75th and 25th percentile of expression to help standardize differences in expression levels by miRNA. Assessment of miRNAs associated to apoptosis genes, utilized available data from 1134 colon cancer cases and 721 rectal cancer cases. This allowed us to analyze survival with colon and rectal cancer separately.

\section{Bioinformatics analysis}

We assessed seed-region matches between mRNAs and miRNAs with FC of $<0.67$ or $>1.50$ that were statistically significant as described in our previous work [21]. We included seeds of six, seven, and eight nucleotides in length when determining seed-region matches. We believe that a seed match would increase the likelihood that an identified miRNA:mRNA interaction having a greater likelihood of a direct biological effect on expression since there are more likely to bind. Of particular importance are those mRNA:miRNA associations where the differential expression of one (either mRNA or miRNA) is inversely associated with the differential expression of the other. These associations are shown by a negative beta coefficient from the linear regression analysis. We used FASTA sequences generated from both GRCh37 and GRCh38 Homo sapiens, using UCSC Table Browser (https://genome.ucsc.edu/cgibin/hgTables) [22]. Detailed methods have been previously described [13, 14, 21].

\section{Results}

The study population consisted of individuals who were predominately diagnosed with colon cancer $(77.9 \%)$, were male $(54.4 \%)$, were non-Hispanic white $(74.2 \%)$, and had MSS tumors (86.6\%) (Table 1). At the end of follow-up the majority of the population was alive (57.4\%).

Of the 133 genes analyzed, 23 were significantly downregulated with a $\mathrm{FC}<0.67$ and 18 were significantly upregulated with a FC $>1.5$ (Table 2). Five genes, SPTA1, $M A P K 10, T U B A L 3, C S F 2 R B$, and BCL2 all had a FC of $<0.4$ and nine genes, $L M N B 1$, TUBA1C, PTPN13, LMNB2, BCL2L1, GZMB, BIRC5, PMAIP1, and CTSL2, had a FC of $>2.0$. Of the genes that were significantly differentially expressed in all CRC tumors combined, all but TUBA3E, were not downregulated in MSS tumors with a FC of $<0.67$, and several genes (NTRK1, FAS, CASP10, TUBA8, CTSS, FASLG, BLS2L11, LMNA, MAP3K14, BIRC3, and $E I F 2 A K 3$ ) were not downregulated in MSI tumors with a FC of $<0.67$, although power was more limited when examined MSI-specific associations. However, two genes, TNFSF 10 and EIF2AK3 (FCs 0.64) had a greater difference between carcinoma and normal mucosa in MSS-specific tumors (See Supplemental Table 2 for MSS-specific tumors), and three $C T S F$, had greater difference in MSI tumors (FC 0.44). Genes that were upregulated in all CRC combined were for the most part also upregulated to a similar degree in MSS-specific tumors, with the exception of TNFRSF10B $\left(\mathrm{FC}_{\mathrm{all}}=1.51, \mathrm{FC}_{\mathrm{MSS}}=1.48\right)$ and $C T S H\left(\mathrm{FC}_{\mathrm{all}}=1.48, \mathrm{FC}_{\mathrm{MSS}}\right.$ 1.59), which were slightly different FCs in MSS-specific tumors. As for genes that were downregulated, there were 
Table 1 Description of study population

\begin{tabular}{|c|c|c|}
\hline & $\mathrm{N}$ & $\%$ \\
\hline \multicolumn{3}{|l|}{ Site } \\
\hline Colon & 169 & 77.9 \\
\hline Rectal & 48 & 22.1 \\
\hline \multicolumn{3}{|l|}{ Sex } \\
\hline Male & 118 & 54.4 \\
\hline Female & 99 & 45.6 \\
\hline \multicolumn{3}{|l|}{ Age } \\
\hline Mean (SD) & 64.8 & 10.1 \\
\hline \multicolumn{3}{|l|}{ Race } \\
\hline Non-Hispanic White & 161 & 74.2 \\
\hline Hispanic & 14 & 6.5 \\
\hline Non-Hispanic Black & 8 & 3.7 \\
\hline Unknown & 34 & 15.7 \\
\hline \multicolumn{3}{|l|}{ AJCC stage } \\
\hline 1 & 58 & 27.1 \\
\hline 2 & 61 & 28.5 \\
\hline 3 & 72 & 33.6 \\
\hline 4 & 23 & 10.8 \\
\hline \multicolumn{3}{|l|}{ Tumor phenotype } \\
\hline MSS & 187 & 86.6 \\
\hline MSI & 29 & 13.4 \\
\hline \multicolumn{3}{|l|}{ Vital status } \\
\hline Dead & 92 & 42.6 \\
\hline Alive & 124 & 57.4 \\
\hline
\end{tabular}

more differences in MSI-specific tumors than for genes that were upregulated. Four genes, PARP4, AIFM1, CTSK, and $G Z M B$, were not upregulated $>1.50$ for MSI tumors, while, DDIT3, ACTG1, CTSL1, HRAS, and TNFRSF10D, were more strongly upregulated in MSI-specific tumors (FCs $1.53,1.55,1.59,1.92$, and 1.503 respectively) (See Supplemental Table 3 for MSI-specific tumors). Figure 1 shows the up and downregulated genes in the KEGG Apoptosis Pathway. Genes that were specifically altered for only MSS and MSI carcinomas suggest unique tumor phenotype and disease pathways, whereas those seen for MSS, MSI and all CRC carcinomas suggest a broader application.

Of the 41 genes with a FC of $<0.67$ or $>1.50$ analyzed with miRNA differential expression, 11 were significantly associated after adjustment for multiple comparisons (Table 3). There were a total of 48 miRNA:mRNA associations with BIRC5 having the greatest number of miRNA associations. Ten of the 14 miRNAs associated with BIRC5 has a seed-region match, and four of these matches, miR145-5p, miR-150-5p, miR-195-5p, and miR-650, had a negative beta coefficient. CSF $2 R B$ was associated with ten miRNAs, five of which had a seed-region match, and one miRNA, miR-92a-3p, had a negative beta coefficient. Two miRNAs, miR-20b-5p, and miR-501-3p associated with
CTSS had both a seed-region match and a negative beta coefficient between the differentially expressed gene and the differentially expressed miRNA. Other genes and miRNA pairs that had both a seed-region match and a negative beta coefficient were TNFRSF10 with miR-196b-5p, CASP7 with miR29b-3p, and BCL2 with miR-203a. Several miRNAs were associated with multiple genes, including miR-150-5p with six genes (BIRC5, CSF2RF, TUBAIB, IRPRI, PIK3CD, and $B C L 2)$, miR-650 with five genes (BIRC5, CSF2RB, ITPR1, $P I K 3 C D$, and BCL2), miR-195-5p with three genes (BIRC5, $T U B 1 B$, and BCL2), and miR-20b-5p and miR-3124-5p each with two genes (miR-20b-5p with BIRC5 and CTSS and miR-3124-5p with TNFRSF10B and CSF2RB).

There were no significant associations between differentially expressed mRNAs and survival. However, evaluation of those miRNAs associated with differentially expressed genes with survival, showed that 11 genes were associated with survival with both significant raw $\mathrm{p}$ values and Q-values less than 0.05 (Table 4). Increased miRNA expression of miR-124-3p, miR-145-3p, miR-193b-3p, and miR-934 in carcinomas was associated with worse survival while increased expression of other miRNAs (i.e. miR-17-5p, miR19b-3p, miR-20a-5p, miR-20b-5p, miR-425-5p, miR-92a-3p, and miR-93-5p) in carcinoma tissue improved survival. Four miRNAs were associated with survival after being diagnosed with colon cancer, miR-124-3p, miR-145-5p, miR-193b-3p, and miR-934, although none of these associations remained significant after adjustment for multiple comparisons. However, 16 miRNAs were associated with survival after a diagnosis with rectal cancer after adjustment for multiple comparisons. Ten of these miRNAs had seed-region matches and six of these miRNAs, miR-150-5p, miR-196b-5p, miR-203a, miR-20b-5p, miR-501-3p, and miR-92a-3p, had negative beta coefficients between differentially expressed miRNA and mRNA, suggesting a greater likelihood for direct binding that would alter the gene expression. In all instances, having greater expression of miRNA in the carcinoma than in the normal mucosa resulted in improved survival.

\section{Discussion}

Our data suggest that miRNAs are involved in apoptosis at several key junctions that involve both the extrinsic pathway, given associations with TRAIL-R (TNFRSF 1OB) and IP3R, $B I R C 5$, and $C A S P 7$, as well as with the intrinsic pathway via associations with Cathepsin and members of the Bcl-2 family and inflammation via CSF2RB (IL3RB) and PI3K. Several miRNAs associated with this pathway also were associated with survival. Our findings identify key points within the pathway that may be suitable for further research and potential targets for therapeutic intervention. 
Table 2 Differentially expressed genes in the KEGG Apoptosis Pathway

\begin{tabular}{|c|c|c|c|c|c|}
\hline Gene name & Tumor mean & Normal mean & Fold change & $\mathrm{p}$ value & Adjusted $\mathrm{p}$ value \\
\hline SPTAl & 0.73 & 2.32 & 0.32 & $1.73 \mathrm{E}-06$ & $3.03 \mathrm{E}-06$ \\
\hline МАР 10 & 9.26 & 28.10 & 0.33 & $8.93 \mathrm{E}-34$ & $1.48 \mathrm{E}-32$ \\
\hline TUBAL3 & 3.06 & 8.27 & 0.37 & $4.43 \mathrm{E}-16$ & $1.44 \mathrm{E}-15$ \\
\hline$C S F 2 R B$ & 27.82 & 73.57 & 0.38 & $7.06 \mathrm{E}-38$ & $1.88 \mathrm{E}-36$ \\
\hline$B C L 2$ & 24.42 & 62.11 & 0.39 & $3.73 E-39$ & $1.24 \mathrm{E}-37$ \\
\hline FOS & 185.52 & 453.52 & 0.41 & $9.65 \mathrm{E}-31$ & $1.07 \mathrm{E}-29$ \\
\hline CASP12 & 0.42 & 0.94 & 0.45 & $7.00 \mathrm{E}-04$ & $1.02 \mathrm{E}-03$ \\
\hline NTRK1 & 1.10 & 2.43 & 0.45 & $3.24 \mathrm{E}-07$ & $5.98 \mathrm{E}-07$ \\
\hline ITPR1 & 57.21 & 109.94 & 0.52 & $5.07 \mathrm{E}-36$ & $9.63 \mathrm{E}-35$ \\
\hline$F A S$ & 28.16 & 50.95 & 0.55 & $2.75 \mathrm{E}-24$ & $1.66 \mathrm{E}-23$ \\
\hline CASP1O & 65.13 & 111.98 & 0.58 & $5.20 \mathrm{E}-32$ & $6.92 \mathrm{E}-31$ \\
\hline$P I K 3 C D$ & 27.33 & 46.48 & 0.59 & $9.96 \mathrm{E}-22$ & $4.42 \mathrm{E}-21$ \\
\hline TUBA 8 & 3.28 & 5.46 & 0.60 & $8.69 \mathrm{E}-09$ & $1.75 \mathrm{E}-08$ \\
\hline$I L 3 R A$ & 2.21 & 3.59 & 0.62 & $2.82 \mathrm{E}-03$ & $3.95 \mathrm{E}-03$ \\
\hline CASP7 & 59.81 & 96.30 & 0.62 & $1.52 \mathrm{E}-24$ & $1.01 \mathrm{E}-23$ \\
\hline$T U B A 3 E$ & 0.42 & 0.68 & 0.62 & $9.17 \mathrm{E}-03$ & $1.22 \mathrm{E}-02$ \\
\hline CTSS & 113.76 & 180.97 & 0.63 & $1.46 \mathrm{E}-23$ & $7.76 \mathrm{E}-23$ \\
\hline$F A S L G$ & 1.07 & 1.70 & 0.63 & $4.96 \mathrm{E}-03$ & $6.74 \mathrm{E}-03$ \\
\hline$B C L 2 L 11$ & 56.19 & 88.47 & 0.64 & $1.23 \mathrm{E}-23$ & $6.82 \mathrm{E}-23$ \\
\hline$L M N A$ & 245.65 & 386.57 & 0.64 & $3.99 \mathrm{E}-20$ & $1.66 \mathrm{E}-19$ \\
\hline МАР $3 K 14$ & 33.86 & 51.73 & 0.65 & $1.46 \mathrm{E}-24$ & $1.01 \mathrm{E}-23$ \\
\hline BIRC3 & 79.53 & 120.36 & 0.66 & $1.83 \mathrm{E}-14$ & $5.17 \mathrm{E}-14$ \\
\hline$E I F 2 A K 3$ & 52.04 & 78.19 & 0.67 & $3.08 \mathrm{E}-23$ & $1.57 \mathrm{E}-22$ \\
\hline МАРКЗ & 62.34 & 92.73 & 0.67 & $9.12 \mathrm{E}-19$ & $3.37 \mathrm{E}-18$ \\
\hline TNFSF 10 & 48.33 & 71.22 & 0.68 & $1.22 \mathrm{E}-12$ & $3.07 \mathrm{E}-12$ \\
\hline CFLAR & 203.49 & 290.53 & 0.70 & $2.89 \mathrm{E}-29$ & $2.96 \mathrm{E}-28$ \\
\hline$C T S W$ & 2.82 & 3.99 & 0.71 & $4.76 \mathrm{E}-04$ & $7.11 \mathrm{E}-04$ \\
\hline$R I P K 1$ & 49.35 & 69.68 & 0.71 & $1.19 \mathrm{E}-19$ & $4.53 \mathrm{E}-19$ \\
\hline ERN1 & 27.13 & 38.17 & 0.71 & $4.23 \mathrm{E}-11$ & $9.87 \mathrm{E}-11$ \\
\hline$B A D$ & 13.13 & 18.42 & 0.71 & $1.38 \mathrm{E}-11$ & $3.28 \mathrm{E}-11$ \\
\hline CAPN2 & 244.01 & 340.39 & 0.72 & $1.09 \mathrm{E}-22$ & $5.38 \mathrm{E}-22$ \\
\hline CASP9 & 13.05 & 17.74 & 0.74 & $1.52 \mathrm{E}-08$ & $3.01 \mathrm{E}-08$ \\
\hline TP53AIP1 & 0.61 & 0.81 & 0.75 & $1.59 \mathrm{E}-01$ & $1.87 \mathrm{E}-01$ \\
\hline TNFRSF1A & 90.65 & 118.74 & 0.76 & $4.32 \mathrm{E}-20$ & $1.74 \mathrm{E}-19$ \\
\hline ATM & 239.32 & 312.70 & 0.77 & $4.82 \mathrm{E}-16$ & $1.52 \mathrm{E}-15$ \\
\hline$D A B 2 I P$ & 144.00 & 186.15 & 0.77 & $1.71 \mathrm{E}-14$ & $4.95 \mathrm{E}-14$ \\
\hline MCL1 & 615.27 & 794.46 & 0.77 & $3.54 \mathrm{E}-14$ & $9.82 \mathrm{E}-14$ \\
\hline CYCS & 95.12 & 122.36 & 0.78 & $1.03 \mathrm{E}-10$ & $2.35 \mathrm{E}-10$ \\
\hline NFKBIA & 58.77 & 75.03 & 0.78 & $1.13 \mathrm{E}-07$ & $2.17 \mathrm{E}-07$ \\
\hline AKT3 & 41.45 & 52.56 & 0.79 & $2.51 \mathrm{E}-05$ & $4.28 \mathrm{E}-05$ \\
\hline$C T S F$ & 7.12 & 8.89 & 0.80 & $1.10 \mathrm{E}-02$ & $1.44 \mathrm{E}-02$ \\
\hline$B A K 1$ & 20.05 & 24.24 & 0.83 & $2.77 \mathrm{E}-05$ & $4.60 \mathrm{E}-05$ \\
\hline$C T S D$ & 270.28 & 324.99 & 0.83 & $4.20 \mathrm{E}-07$ & $7.66 \mathrm{E}-07$ \\
\hline$B I R C 2$ & 81.92 & 98.12 & 0.83 & $6.71 \mathrm{E}-10$ & $1.44 \mathrm{E}-09$ \\
\hline TRADD & 25.11 & 28.92 & 0.87 & $2.47 \mathrm{E}-03$ & $3.53 \mathrm{E}-03$ \\
\hline ITPR2 & 221.72 & 255.25 & 0.87 & $3.28 \mathrm{E}-03$ & $4.55 \mathrm{E}-03$ \\
\hline$G A D D 45 G$ & 2.79 & 3.18 & 0.88 & $2.07 \mathrm{E}-01$ & $2.39 \mathrm{E}-01$ \\
\hline$R A F 1$ & 131.53 & 147.29 & 0.89 & $5.78 \mathrm{E}-07$ & $1.04 \mathrm{E}-06$ \\
\hline TRAFI & 46.52 & 51.87 & 0.90 & $1.55 \mathrm{E}-02$ & $1.98 \mathrm{E}-02$ \\
\hline$K R A S$ & 107.64 & 117.88 & 0.91 & $1.65 \mathrm{E}-02$ & $2.09 \mathrm{E}-02$ \\
\hline$M A P 2 K 2$ & 69.97 & 75.76 & 0.92 & $1.47 \mathrm{E}-02$ & $1.90 \mathrm{E}-02$ \\
\hline
\end{tabular}


Table 2 (continued)

\begin{tabular}{|c|c|c|c|c|c|}
\hline Gene name & Tumor mean & Normal mean & Fold change & $\mathrm{p}$ value & Adjusted $\mathrm{p}$ value \\
\hline$N F K B 1$ & 77.63 & 83.98 & 0.92 & $7.65 \mathrm{E}-03$ & $1.03 \mathrm{E}-02$ \\
\hline МAP $3 K 5$ & 68.22 & 73.59 & 0.93 & $3.82 \mathrm{E}-02$ & $4.71 \mathrm{E}-02$ \\
\hline$P I D D$ & 28.99 & 31.19 & 0.93 & $1.08 \mathrm{E}-01$ & $1.30 \mathrm{E}-01$ \\
\hline$P A R P 3$ & 18.95 & 20.17 & 0.94 & $2.44 \mathrm{E}-01$ & $2.78 \mathrm{E}-01$ \\
\hline$I K B K B$ & 137.36 & 145.80 & 0.94 & $6.63 \mathrm{E}-02$ & $8.08 \mathrm{E}-02$ \\
\hline$A P A F 1$ & 85.50 & 90.73 & 0.94 & $7.64 \mathrm{E}-02$ & $9.24 \mathrm{E}-02$ \\
\hline$P A R P 2$ & 20.40 & 21.49 & 0.95 & $3.35 \mathrm{E}-01$ & $3.68 \mathrm{E}-01$ \\
\hline SEPT4 & 6.07 & 6.38 & 0.95 & $5.52 \mathrm{E}-01$ & $5.78 \mathrm{E}-01$ \\
\hline$T N F$ & 1.95 & 2.04 & 0.96 & $7.52 \mathrm{E}-01$ & $7.58 \mathrm{E}-01$ \\
\hline CASP3 & 42.94 & 44.90 & 0.96 & $3.39 \mathrm{E}-01$ & $3.69 \mathrm{E}-01$ \\
\hline CTSC & 83.85 & 87.45 & 0.96 & $2.63 \mathrm{E}-01$ & $2.93 \mathrm{E}-01$ \\
\hline TNFRSF1OD & 21.48 & 22.33 & 0.96 & $5.20 \mathrm{E}-01$ & $5.49 \mathrm{E}-01$ \\
\hline$T U B A 3 D$ & 2.86 & 2.96 & 0.97 & $7.43 \mathrm{E}-01$ & $7.55 \mathrm{E}-01$ \\
\hline PRF1 & 5.19 & 5.24 & 0.99 & $9.15 \mathrm{E}-01$ & $9.15 \mathrm{E}-01$ \\
\hline$A K T 1$ & 153.83 & 152.34 & 1.01 & $7.08 \mathrm{E}-01$ & $7.24 \mathrm{E}-01$ \\
\hline$J U N$ & 214.48 & 211.03 & 1.02 & $7.02 \mathrm{E}-01$ & $7.24 \mathrm{E}-01$ \\
\hline MAPK 8 & 62.01 & 60.98 & 1.02 & $6.19 \mathrm{E}-01$ & $6.43 \mathrm{E}-01$ \\
\hline$M A P K 1$ & 183.50 & 179.79 & 1.02 & $3.53 \mathrm{E}-01$ & $3.78 \mathrm{E}-01$ \\
\hline МАРК & 65.92 & 64.12 & 1.03 & $4.12 \mathrm{E}-01$ & $4.39 \mathrm{E}-01$ \\
\hline PIK3R1 & 154.42 & 149.09 & 1.04 & $3.00 \mathrm{E}-01$ & $3.32 \mathrm{E}-01$ \\
\hline PIK3R3 & 35.88 & 33.87 & 1.06 & $2.55 \mathrm{E}-01$ & $2.87 \mathrm{E}-01$ \\
\hline CASP6 & 22.61 & 21.28 & 1.06 & $2.10 \mathrm{E}-01$ & $2.41 \mathrm{E}-01$ \\
\hline$G A D D 45 B$ & 12.90 & 12.05 & 1.07 & $3.43 \mathrm{E}-01$ & $3.71 \mathrm{E}-01$ \\
\hline$P D P K 1$ & 100.97 & 93.03 & 1.09 & $3.80 \mathrm{E}-03$ & $5.21 \mathrm{E}-03$ \\
\hline$I K B K G$ & 6.91 & 6.20 & 1.11 & $2.04 \mathrm{E}-01$ & $2.38 \mathrm{E}-01$ \\
\hline$P I K 3 C B$ & 90.89 & 81.36 & 1.12 & $5.56 \mathrm{E}-04$ & $8.21 \mathrm{E}-04$ \\
\hline$R E L A$ & 82.20 & 73.32 & 1.12 & $5.56 \mathrm{E}-06$ & $9.60 \mathrm{E}-06$ \\
\hline CTSZ & 123.07 & 109.77 & 1.12 & $2.59 \mathrm{E}-03$ & $3.66 \mathrm{E}-03$ \\
\hline ITPR3 & 420.93 & 374.92 & 1.12 & $2.74 \mathrm{E}-05$ & $4.60 \mathrm{E}-05$ \\
\hline$D I A B L O$ & 39.85 & 35.12 & 1.13 & $6.78 \mathrm{E}-05$ & $1.07 \mathrm{E}-04$ \\
\hline$C A P N 1$ & 187.82 & 164.50 & 1.14 & $1.79 \mathrm{E}-07$ & $3.41 \mathrm{E}-07$ \\
\hline ENDOG & 12.68 & 11.09 & 1.14 & $2.72 \mathrm{E}-02$ & $3.41 \mathrm{E}-02$ \\
\hline$G A D D 45 A$ & 13.07 & 11.37 & 1.15 & $3.07 \mathrm{E}-02$ & $3.81 \mathrm{E}-02$ \\
\hline$A K T 2$ & 156.81 & 135.87 & 1.15 & $2.66 \mathrm{E}-07$ & $4.99 \mathrm{E}-07$ \\
\hline SPTAN1 & 543.11 & 467.35 & 1.16 & $3.33 \mathrm{E}-10$ & $7.38 \mathrm{E}-10$ \\
\hline$M A P 2 K 1$ & 32.75 & 28.06 & 1.17 & $4.28 \mathrm{E}-04$ & $6.55 \mathrm{E}-04$ \\
\hline CTSL1 & 20.11 & 17.18 & 1.17 & $1.18 \mathrm{E}-02$ & $1.53 \mathrm{E}-02$ \\
\hline CHUK & 44.48 & 37.81 & 1.18 & $4.50 \mathrm{E}-04$ & $6.80 \mathrm{E}-04$ \\
\hline HTRA2 & 19.32 & 16.19 & 1.19 & $5.92 \mathrm{E}-05$ & $9.61 \mathrm{E}-05$ \\
\hline XIAP & 187.63 & 156.09 & 1.20 & $8.16 \mathrm{E}-13$ & $2.13 \mathrm{E}-12$ \\
\hline PIK3CA & 67.11 & 55.23 & 1.22 & $6.77 \mathrm{E}-07$ & $1.20 \mathrm{E}-06$ \\
\hline$F A D D$ & 14.64 & 12.03 & 1.22 & $7.19 \mathrm{E}-04$ & $1.04 \mathrm{E}-03$ \\
\hline$B A X$ & 36.23 & 29.75 & 1.22 & $1.59 \mathrm{E}-04$ & $2.49 \mathrm{E}-04$ \\
\hline CASP8 & 70.64 & 57.92 & 1.22 & $3.90 \mathrm{E}-09$ & $8.11 \mathrm{E}-09$ \\
\hline$D F F B$ & 16.02 & 13.07 & 1.23 & $1.87 \mathrm{E}-04$ & $2.89 \mathrm{E}-04$ \\
\hline ATF4 & 130.84 & 105.67 & 1.24 & $8.92 \mathrm{E}-13$ & $2.28 \mathrm{E}-12$ \\
\hline$B C L 2 A 1$ & 2.01 & 1.61 & 1.25 & $1.59 \mathrm{E}-01$ & $1.87 \mathrm{E}-01$ \\
\hline$D A X X$ & 35.66 & 28.09 & 1.27 & $3.63 \mathrm{E}-10$ & $7.92 \mathrm{E}-10$ \\
\hline$B I D$ & 35.72 & 27.95 & 1.28 & $2.19 \mathrm{E}-09$ & $4.63 \mathrm{E}-09$ \\
\hline TNFRSF10A & 37.99 & 28.64 & 1.33 & $2.17 \mathrm{E}-08$ & $4.25 \mathrm{E}-08$ \\
\hline PIK3R2 & 72.83 & 54.55 & 1.34 & $1.32 \mathrm{E}-18$ & $4.73 \mathrm{E}-18$ \\
\hline
\end{tabular}


Table 2 (continued)

\begin{tabular}{|c|c|c|c|c|c|}
\hline Gene name & Tumor mean & Normal mean & Fold change & $\mathrm{p}$ value & Adjusted $\mathrm{p}$ value \\
\hline DDIT3 & 11.07 & 8.29 & 1.34 & $3.01 \mathrm{E}-05$ & $4.93 \mathrm{E}-05$ \\
\hline$A C T B$ & 2123.22 & 1584.09 & 1.34 & $3.58 \mathrm{E}-28$ & $3.18 \mathrm{E}-27$ \\
\hline$N R A S$ & 104.64 & 76.74 & 1.36 & $3.26 \mathrm{E}-15$ & $9.64 \mathrm{E}-15$ \\
\hline$T R A F 2$ & 40.20 & 29.46 & 1.36 & $8.02 \mathrm{E}-13$ & $2.13 \mathrm{E}-12$ \\
\hline$P A R P 1$ & 109.63 & 79.73 & 1.37 & $5.66 \mathrm{E}-16$ & $1.75 \mathrm{E}-15$ \\
\hline EIF $2 S 1$ & 70.00 & 50.27 & 1.39 & $6.06 \mathrm{E}-16$ & $1.83 \mathrm{E}-15$ \\
\hline$D F F A$ & 86.27 & 61.10 & 1.41 & $2.64 \mathrm{E}-24$ & $1.66 \mathrm{E}-23$ \\
\hline TUBA1A & 27.86 & 19.65 & 1.42 & $8.44 \mathrm{E}-09$ & $1.73 \mathrm{E}-08$ \\
\hline$C A S P 2$ & 124.24 & 87.36 & 1.42 & $9.53 \mathrm{E}-25$ & $7.04 \mathrm{E}-24$ \\
\hline$C T S B$ & 513.97 & 360.55 & 1.43 & $2.50 \mathrm{E}-22$ & $1.19 \mathrm{E}-21$ \\
\hline ACTG1 & 1209.84 & 823.20 & 1.47 & $6.71 \mathrm{E}-29$ & $6.38 \mathrm{E}-28$ \\
\hline $\mathrm{CTSH}$ & 76.75 & 51.92 & 1.48 & $5.16 \mathrm{E}-14$ & $1.40 \mathrm{E}-13$ \\
\hline$H R A S$ & 18.52 & 12.37 & 1.50 & $3.52 \mathrm{E}-12$ & $8.66 \mathrm{E}-12$ \\
\hline PARP4 & 340.90 & 226.33 & 1.51 & $2.95 \mathrm{E}-26$ & $2.45 \mathrm{E}-25$ \\
\hline TNFRSF1OB & 135.53 & 89.65 & 1.51 & $3.45 \mathrm{E}-21$ & $1.48 \mathrm{E}-20$ \\
\hline AIFM1 & 41.19 & 27.11 & 1.52 & $4.56 \mathrm{E}-17$ & $1.55 \mathrm{E}-16$ \\
\hline$C T S K$ & 48.58 & 31.12 & 1.56 & $8.29 \mathrm{E}-12$ & $2.00 \mathrm{E}-11$ \\
\hline TNFRSF10C & 3.54 & 2.10 & 1.69 & $6.25 \mathrm{E}-05$ & $1.00 \mathrm{E}-04$ \\
\hline$T U B A 4 A$ & 33.42 & 19.75 & 1.69 & $3.16 \mathrm{E}-22$ & $1.45 \mathrm{E}-21$ \\
\hline TP53 & 105.07 & 59.63 & 1.76 & $3.25 \mathrm{E}-24$ & $1.88 \mathrm{E}-23$ \\
\hline$B B C 3$ & 20.58 & 11.49 & 1.79 & $2.02 \mathrm{E}-18$ & $7.06 \mathrm{E}-18$ \\
\hline$T U B A 1 B$ & 138.84 & 76.60 & 1.81 & $3.01 \mathrm{E}-31$ & $3.64 \mathrm{E}-30$ \\
\hline$L M N B 1$ & 89.36 & 43.88 & 2.04 & $1.01 \mathrm{E}-36$ & $2.23 \mathrm{E}-35$ \\
\hline TUBAIC & 69.89 & 33.41 & 2.09 & $1.39 \mathrm{E}-32$ & $2.05 \mathrm{E}-31$ \\
\hline PTPN13 & 69.58 & 32.80 & 2.12 & $4.54 \mathrm{E}-20$ & $1.78 \mathrm{E}-19$ \\
\hline$L M N B 2$ & 150.48 & 68.95 & 2.18 & $1.08 \mathrm{E}-45$ & $7.20 \mathrm{E}-44$ \\
\hline$B C L 2 L 1$ & 144.28 & 64.05 & 2.25 & $1.06 \mathrm{E}-56$ & $1.41 \mathrm{E}-54$ \\
\hline$G Z M B$ & 4.21 & 1.49 & 2.83 & $2.11 \mathrm{E}-10$ & $4.77 \mathrm{E}-10$ \\
\hline BIRC5 & 35.63 & 11.31 & 3.15 & $1.67 \mathrm{E}-39$ & $7.41 \mathrm{E}-38$ \\
\hline PMAIP1 & 11.00 & 3.31 & 3.32 & $6.06 \mathrm{E}-26$ & $4.74 \mathrm{E}-25$ \\
\hline CTSL2 & 4.38 & 1.21 & 3.64 & $8.07 \mathrm{E}-17$ & $2.68 \mathrm{E}-16$ \\
\hline
\end{tabular}

By utilizing seed-region matches we were able to identify miRNAs that were more likely to have a direct biological effect on the mRNA that could in turn influence the apoptosis pathway. MiRNA binding with the 3'UTR of the mRNA increases the likelihood of mRNA degradation; this is especially relevant when increases in differential expression of miRNA results in decreases in differential expression of mRNA. This suggests that the miRNA is directly influencing the mRNA expression and may represent an area in the pathway where miRNAs can directly exert influence on the apoptotic process. Indirect effects are most likely operating feedback and feed-forward loops [23-25]. In feed-back loops, regulators such as miRNAs and transcription factors (TFs) can have either the same effect (repression of expression) or opposite effects where the TF enhances the mRNA [24]. In feed-forward loops, TF regulates the miRNA as well as a target gene (TG), which is in turn also regulated by the miRNA. In this instance, the miRNA may regulate the TG directly, through seed region binding leading to mRNA degradation or translational repression, or indirectly, through repression of the TF that is influencing transcription of the same TG. Regulatory networks involving miRNAs are believed to be prevalent mechanisms for modulating gene expression [24]. While indirect effects are most likely important in the apoptosis pathway, the direct binding between miRNA and mRNA that results in inverse expression associations have a greater potential as a target of future research and is the focus of the discussion of our results.

Cathepsins are a family of "lysosomal proteolytic enzymes" [26] that are released from lysosomes into the cytoplasm, triggering apoptosis. They are involved in both the intrinsic pathway, regulating the release of pro-apoptotic factors from the mitochondria, but also are involved in the extrinsic pathway of apoptosis given the ability to block inhibitors of apoptosis (IAP). In our study both CTSK (cathepsin K) and CTSS (cathepsin S) were associated with 


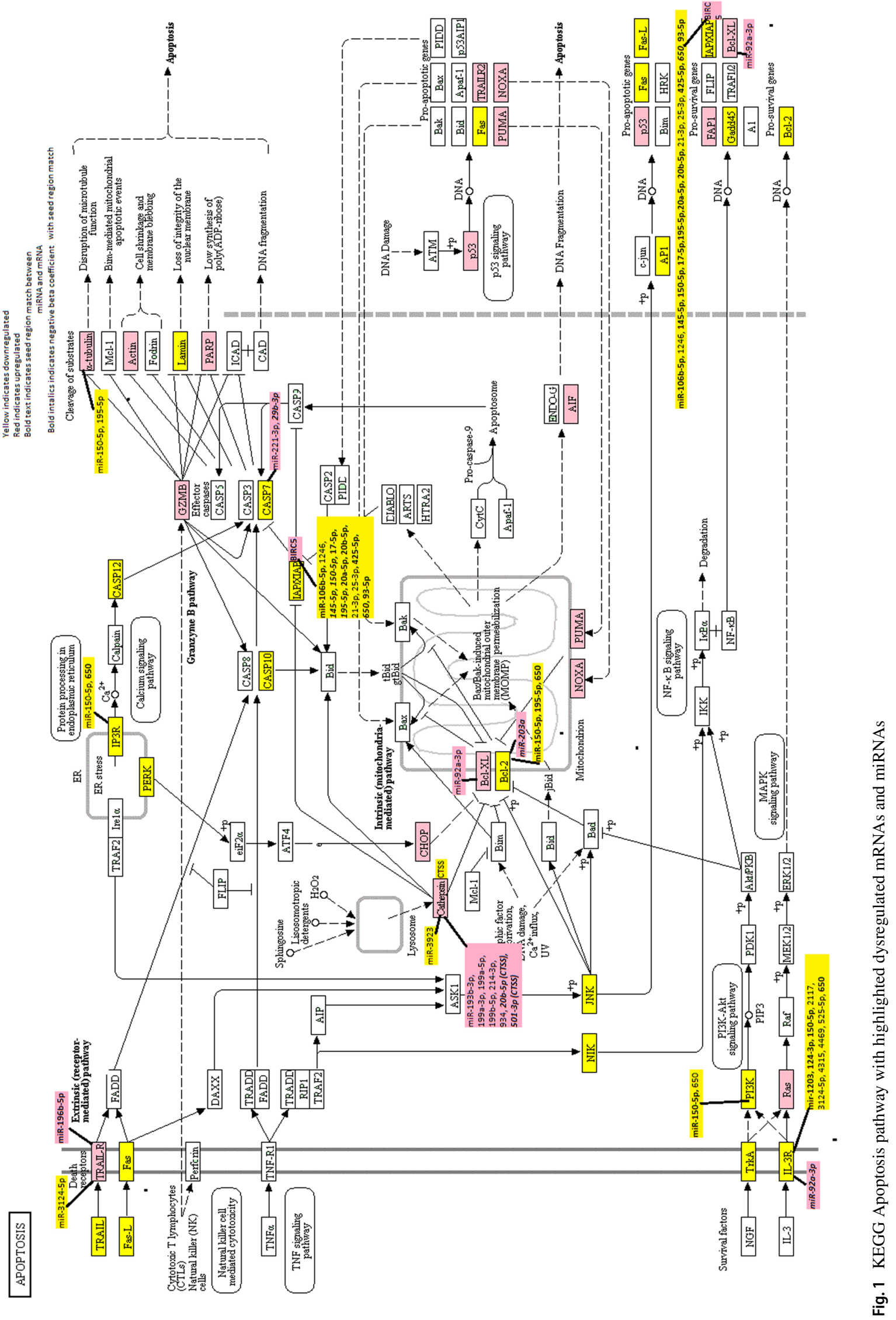




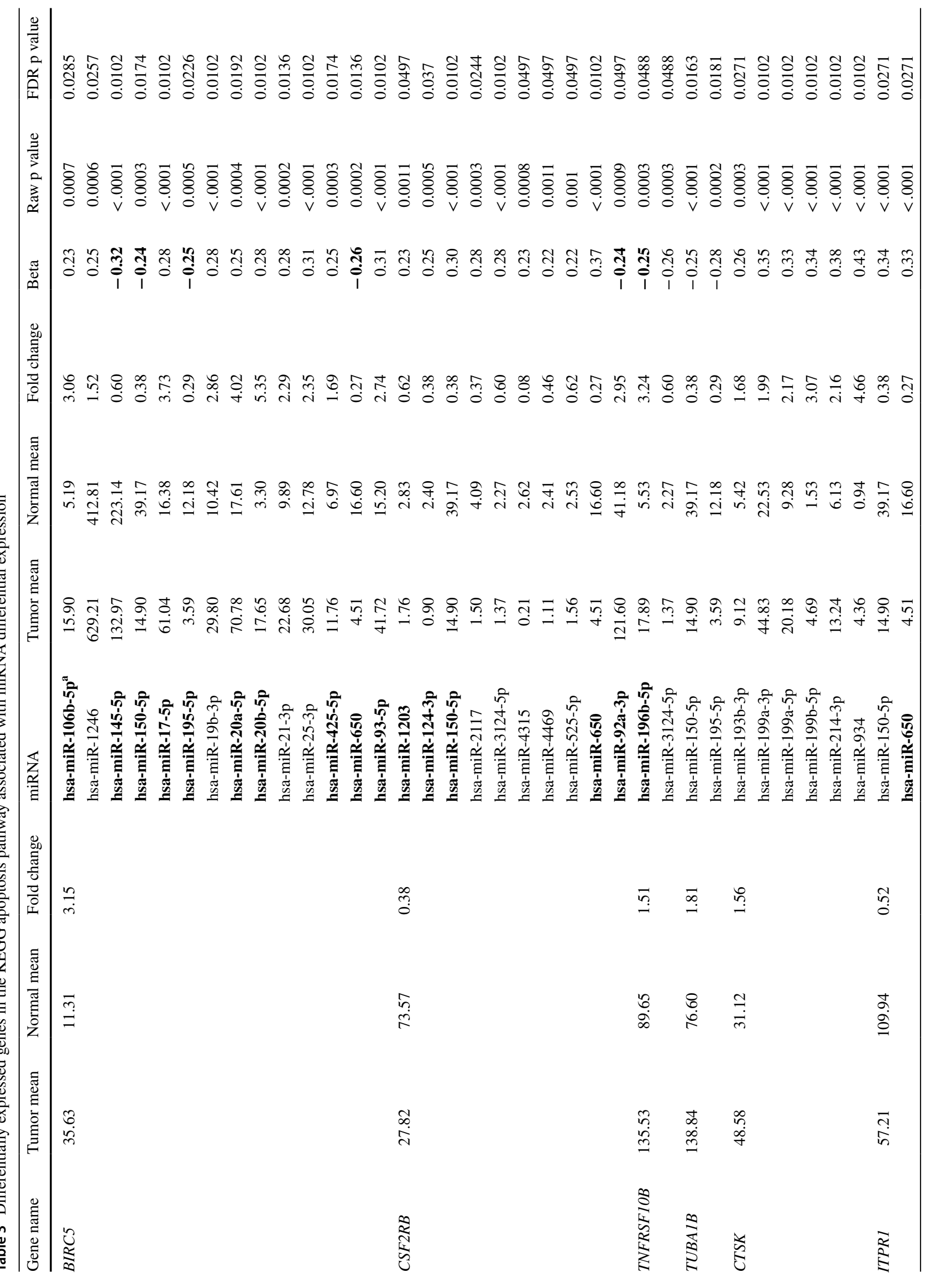




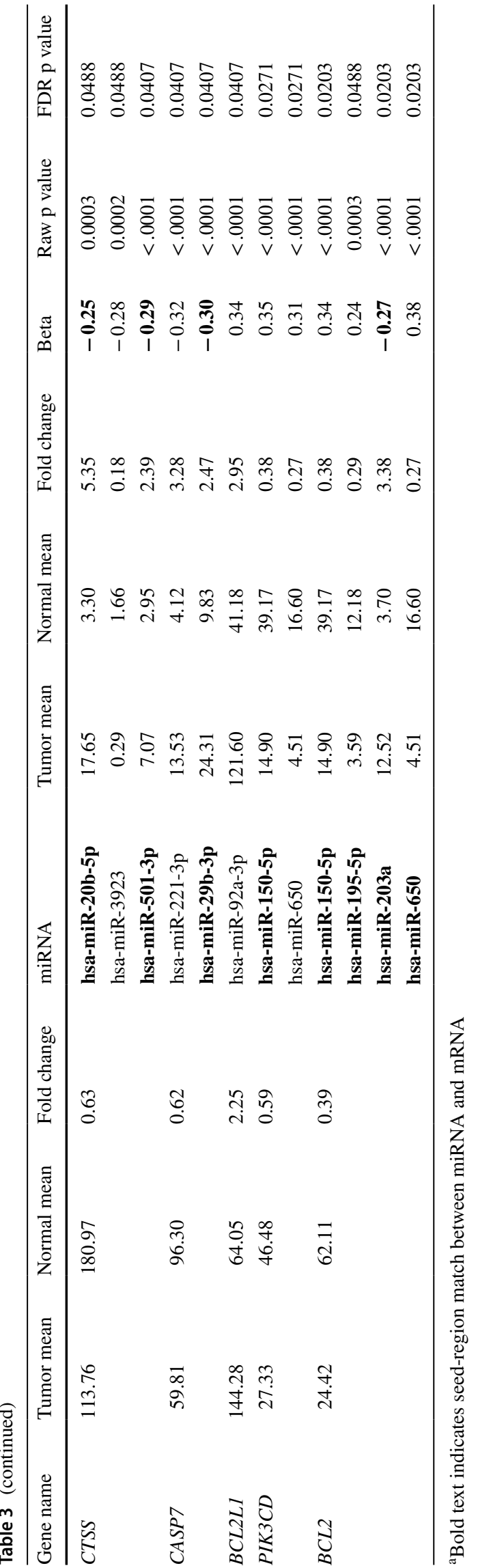

miRNAs, however, only CTSS was directly associated given the likely binding of miR-20b-5p and miR-501-3p that could result in a decreased expression of CTSS in the presence of increased differential expression of these miRNAs. CTSS is a cysteine cathepsin that has been linked previously to tumorigenesis and to response to chemotherapy in CRC patients [27]. Studies have suggested that cathepsin S promotes tumor invasion through extracellular matrix degradation that release matrix-derived growth factors that drive angiogenesis [28]. Cathepsin S can block members of the Bcl-2 family as well as IAPs. Given that CTSS was downregulated, two components of the pathway could be affected. $B C L 2 L 1$, an apoptosis inhibitor, was possibly up-regulated since $C T S S$ could not inhibit it. Another member of the Bcl-2 family, anti-apoptotic $B C L 2$, was down-regulated. $B C L 2$ was directly associated through seed-region match to miR-203a, which could also result in decreased expression of $B C L 2$, which would activate the intrinsic apoptosis pathway. $B L C 2$ has been examined with several targeted miRNAs, including miR-491, miR-143, miR-148a, miR-365, miR-1915, miR204, and miR-125b [4-6, 8, 29-31]. We did not see direct associations with any of these genes, however we could have missed associations since we only examined gene expression data and protein activity could have been altered. We did note that $B C L 2$ had seed-region matches with all of these miRNAs except miR-491, suggesting that associations likely exist but were not detected in this study, possibly because of an effect on protein level rather than on mRNA expression. Also, given our study design and adjustment for multiple comparisons we could have not detected significant associations in our broader study. Likewise, our uniquely identified associations with BCL2 and miRNAs most likely reflects that they have not being previously examined, as much of the literature is based on targeted miRNAs rather than all miRNAs commonly expressed in colorectal tissue [32]. We believe that these miRNAs may be key targets in therapeutics given the apparent role in regulating apoptosis. Our pathway approach to examining miRNAs has hopefully provided insight into unique role of miRNAs as there relate to the carcinogenic process.

In addition to altering the intrinsic pathway, CTSS has a role in blocking IAPs. In our data BIRC5, which encodes for the protein survivin, was one of the most up-regulated genes in the apoptosis pathway. BIRC5 inhibits caspase activity and overexpression of BIRC5 leads to resistance to apoptosis [33]. Survivin blocks apoptosis by binding to caspases, such as $C A S P 7$, an executioner of the apoptosis process that has been explored as a target for cancer therapeutics [34]. In our data, BIRC5 was the gene that had the most seed-region matches with miRNAs, four of which were down-regulated when BIRC5 was up-regulated. The miRNAs that appear to directly target BIRC5 are miR-145-5p, miR-150-5p, miR195-5p, and miR-650; both miR-145-5p and miR-150-5p 
Table 4 Associations between miRNAs associated with KEGG apoptosis pathway and colorectal cancer survival

\begin{tabular}{|c|c|c|c|c|c|c|c|}
\hline miRNA & Q1 & Q3 & $\mathrm{HR}^{\mathrm{a}}$ & $95 \% \mathrm{CI}$ & $\mathrm{p}$ value & $\mathrm{Q}$ value & FDR adjusted $\mathrm{p}$ \\
\hline \multicolumn{8}{|l|}{ All colorectal cancer } \\
\hline hsa-miR-124-3p ${ }^{b}$ & -0.36 & 0.00 & 1.04 & $1.00-1.07$ & 0.04 & 0.04 & 0.14 \\
\hline hsa-miR-145-5p & -1.94 & -0.15 & 1.13 & $1.01-1.26$ & 0.03 & 0.03 & 0.14 \\
\hline hsa-miR-17-5p & 0.95 & 2.40 & 0.91 & $0.84-0.98$ & 0.02 & 0.03 & 0.14 \\
\hline hsa-miR-193b-3p & -0.16 & 1.45 & 1.10 & $1.01-1.20$ & 0.03 & 0.03 & 0.14 \\
\hline hsa-miR-19b-3p & 0.61 & 2.35 & 0.91 & $0.84-0.99$ & 0.02 & 0.03 & 0.14 \\
\hline hsa-miR-20a-5p & 1.02 & 2.61 & 0.91 & $0.84-0.98$ & 0.02 & 0.03 & 0.14 \\
\hline hsa-miR-20b-5p & 0.96 & 3.11 & 0.83 & $0.75-0.91$ & 0.0002 & 0.03 & 0.01 \\
\hline hsa-miR-425-5p & -0.10 & 1.48 & 0.92 & $0.85-0.998$ & 0.04 & 0.04 & 0.14 \\
\hline hsa-miR-92a-3p & 0.63 & 1.87 & 0.91 & $0.83-0.99$ & 0.03 & 0.03 & 0.14 \\
\hline hsa-miR-934 & 0.50 & 2.29 & 1.13 & $1.01-1.27$ & 0.03 & 0.03 & 0.14 \\
\hline hsa-miR-93-5p & 0.71 & 1.99 & 0.93 & $0.86-0.996$ & 0.04 & 0.04 & 0.14 \\
\hline \multicolumn{8}{|l|}{ Colon cancer } \\
\hline hsa-miR-124-3p & -0.36 & 0.00 & 1.05 & $1.01-1.10$ & 0.02 & $\mathrm{NA}^{\mathrm{c}}$ & 0.21 \\
\hline$h s a-m i R-145-5 p$ & -1.88 & -0.03 & 1.18 & $1.03-1.36$ & 0.02 & NA & 0.21 \\
\hline hsa-miR-193b-3p & -0.19 & 1.66 & 1.19 & $1.05-1.34$ & 0.004 & NA & 0.13 \\
\hline \multicolumn{8}{|l|}{ Rectal cancer } \\
\hline hsa-miR-106b-5p & 0.71 & 2.64 & 0.80 & $0.68-0.94$ & 0.01 & 0.06 & 0.03 \\
\hline hsa-miR-1203 & -1.44 & 0.00 & 0.82 & $0.69-0.96$ & 0.02 & 0.06 & 0.04 \\
\hline hsa-miR-150-5p & -2.49 & -0.73 & 0.82 & $0.70-0.96$ & 0.01 & 0.06 & 0.04 \\
\hline hsa-miR-17-5p & 1.13 & 2.43 & 0.80 & $0.70-0.91$ & 0.00 & 0.06 & 0.01 \\
\hline$h s a-m i R-196 b-5 p$ & 0.00 & 3.21 & 0.73 & $0.59-0.89$ & 0.00 & 0.06 & 0.01 \\
\hline hsa-miR-19b-3p & 0.82 & 2.39 & 0.80 & $0.70-0.91$ & 0.00 & 0.06 & 0.01 \\
\hline hsa-miR-203a & 0.00 & 2.76 & 0.82 & $0.68-0.99$ & 0.04 & 0.07 & 0.08 \\
\hline hsa-miR-20a-5p & 1.20 & 2.70 & 0.79 & $0.69-0.91$ & 0.00 & 0.06 & 0.01 \\
\hline$h s a-m i R-20 b-5 p$ & 1.24 & 3.30 & 0.67 & $0.57-0.79$ & 0.00 & 0.06 & 0.00 \\
\hline hsa-miR-21-3p & 0.50 & 1.62 & 0.85 & $0.75-0.97$ & 0.02 & 0.06 & 0.04 \\
\hline hsa-miR-221-3p & 0.63 & 2.57 & 0.81 & $0.69-0.94$ & 0.01 & 0.06 & 0.03 \\
\hline hsa-miR-25-3p & 0.63 & 1.84 & 0.86 & $0.76-0.98$ & 0.02 & 0.06 & 0.05 \\
\hline hsa-miR-425-5p & 0.00 & 1.54 & 0.84 & $0.74-0.95$ & 0.01 & 0.06 & 0.03 \\
\hline hsa-miR-501-3p & 0.57 & 1.73 & 0.81 & $0.69-0.94$ & 0.01 & 0.06 & 0.03 \\
\hline hsa-miR-525-5p & -0.02 & 0.62 & 0.91 & $0.84-0.98$ & 0.01 & 0.06 & 0.04 \\
\hline hsa-miR-92a-3p & 0.78 & 1.86 & 0.78 & $0.67-0.91$ & 0.00 & 0.06 & 0.01 \\
\hline hsa-miR-93-5p & 0.88 & 1.98 & 0.85 & $0.76-0.96$ & 0.01 & 0.06 & 0.03 \\
\hline
\end{tabular}

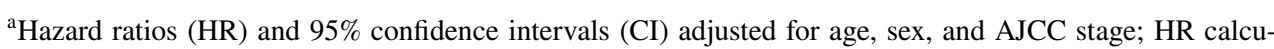
lated as the risk associated with the difference between Q1 and Q3

${ }^{b}$ Bold text indicates seed-region match with mRNA; italics indicates negative beta coefficient between differential expression of mRNA and miRNA for those with a seed-region match

${ }^{\mathrm{c}} \mathrm{Q}$-value not calculated for colon-cancer specific mortality because the estimated number of true null hypothesis is zero were associated with better survival after diagnosed with CRC overall or with rectal cancer specifically when upregulated in CRC tumor tissue. Taken together, these results suggest that decreases in the expression of these miRNAs allowed greater expression of BIRC5 and down-regulation of $C T S S$, resulting in less inhibition of BIRC5 and possibly resistance to apoptosis. When BIRC5 was upregulated, $C A S P 7$ was likely inhibited, as indicated by decreased expression in tumors, as was the predicted effect of a decrease in apoptosis. CASP7 also had a direct biological association with miR-29b-3p, supported by a seed region match and a negative beta coefficient for this association, which would suggest further decreases CASP7 expression. Others have identified miR-203 as being associated with survivin [35], although we did not observe this association we did note that there was a seed-region match between miR203b-5p and BIRC5. The miRNAs identified in our study, miR-145-5p, miR-150-5p, miR-195-5p, miR-650, and miR$29 b-3 p$, may serve as reasonable therapeutic targets given 
their likely role in influencing activity of BIRC5 and CASP7, two important elements in the apoptosis pathway.

A third area of importance in the apoptosis pathway is CSF2RB (Colony Stimulating Factor 2 Beta Common Subset B), or IL-3R, which is a type 1 cytokine receptor. $C S F 2 R B$ was down-regulated (FC 0.38) and associated with 10 miRNAs; miR-92a-3p was up-regulated as $C S F 2 R B$ was down-regulated and there was a seed-region match between the gene and miRNA. Down-regulation of $C S F 2 R B$ could lead to down-regulation of PI3K genes (PIK3CD was downregulated in our data), and alter the MAPK and NFKBsignaling pathways that could ultimately lead to altered expression of genes needed for regulating apoptosis of certain inflammatory cells.

While we did not observe an association between dysregulated genes in the apoptosis pathway and survival, it should be kept in mind that we had limited statistical power to evaluate associations. Power was further limited to evaluate site-specific associations with dysregulated genes. However, evaluation of miRNAs with survival was feasible because for that component of the analysis we had a much larger sample of colon cancer cases and rectal cancer cases with paired miRNA expression data. Our approach was to thus evaluate miRNAs that were associated with mRNAs in the pathway and determine their association with survival. We observed that several miRNAs were associated with CRCspecific survival for colon and rectal cases combined and that the strongest associations were between miRNAs and survival after being diagnosed with rectal cancer. Several of the miRNAs associated with survival appeared to be directly biologically associated with mRNAs, in that an increase in the differential expression of the miRNA reduced the differential mRNA expression. Several of these miRNAs had direct associations with BIRC5, including miR-145-5p and miR-17-5p for all CRC and miR-150-5p and miR-196b-5p for rectal cancer specifically. MiR-20b-5p and miR-501-3p were associated with CTSS, miR-92a-5p was associated with $C S F 2 R B$, and miR-203a was inversely associated with $B C L 2$. These findings suggest that miRNA binding, or lack of binding, results in an increase (i.e. BIRC5) in anti-apoptotic gene expression or a decrease (i.e. CSF2RB, CTSS, and $B C L 2)$ in pro-apoptotic gene expression, which could result in decreased apoptosis, which could favor tumorigenesis and could ultimately influence patient prognosis.

Our sample size is one of the largest available that contains individuals with both carcinoma and normal mucosa expression data. We acknowledge that normal colonic mucosa, while not being entirely normal since it too may have undergone changes from health colonic mucosa, is the closest colonic tissue available for a matched-paired analysis. The normal colonic mucosa utilized was taken from the same colonic site as the tumor; this prevented differences in expression between carcinoma and normal mucosa, from being the result of tumor location. As we stated previously, there are differences in our identified associations from those reported in the literature; this could stem in part from our analysis based on paired samples. A study limitation is our sample size of mRNA data, which would greatly impact our ability to identify associated between dysregulated genes and survival. In interpreting the results of this research it should be acknowledged that $\mathrm{FC}$ cut point for assessment of meaningful changes is arbitrary. Utilization of FC cutpoints of $<0.67$ or $>1.50$ enabled us to focus on changes that might be more biologically meaningful. Small FCs, say of 0.9 or 1.1 , could stem from measurement differences, sample processing, or other factors that lead to small amounts of expression that may not be meaningful. Using these criteria, we examined what we believed to be the most meaningful changes when looking at mRNA:miRNA associations and therefore could have missed other significant associations. We exclusively used the KEGG pathway database to identify apoptosis pathway genes; genes not identified in KEGG but may influence apoptosis as well as influence miRNA expression were not analyzed. Another study limitation is availability of only gene expression data. Since miRNAs may have their impact post-transcriptionally, protein expression data could provide additional insight into associations. However, it should be acknowledged that much of the current information on miRNA target genes comes from gene expression data and associations observed may have important biological meaning $[32,36]$. Studies have been conducted that suggest that even though miRNAs have their impact at the post-transcriptional level, they still commonly alter gene expression $[37,38]$. While conducting more detailed functionality assessment of the interaction between mRNA and miRNA is needed, it is beyond the scope of this study.

In conclusion, our data suggest several key areas in the apoptosis pathway where miRNAs may alter expression of genes such as BIRC5, CASP7, CTSS, CSF2RB, and BCL2, that in turn influences apoptosis and tumorigenesis. The results from this study provide important targets for follow-up in laboratory-based functionality studies that could lead to new cancer therapeutics. We encourage others to replicate these findings and validate their feasibility as therapeutic targets.

Acknowledgements The contents of this manuscript are solely the responsibility of the authors and do not necessarily represent the official view of the National Cancer Institute. We acknowledge Sandra Edwards for data oversight and study management, and Michael Hoffman and Erica Wolff for miRNA analysis. We acknowledge Dr. Bette Caan and the staff at Kaiser Permanente Northern California for sample and data collection. This study was supported by NCI grant CA163683. 


\section{Compliance with ethical standards}

Conflict of interest The authors declare that they have no competing interests.

Open Access This article is distributed under the terms of the Creative Commons Attribution 4.0 International License (http://creativeco mmons.org/licenses/by/4.0/), which permits unrestricted use, distribution, and reproduction in any medium, provided you give appropriate credit to the original author(s) and the source, provide a link to the Creative Commons license, and indicate if changes were made.

\section{References}

1. Elmore S (2007) Apoptosis: a review of programmed cell death. Toxicol Pathol 35(4):495-516

2. Sun Y, Zhao X, Luo M, Zhou Y, Ren W, Wu K, Li X, Shen J, Hu Y (2014) The pro-apoptotic role of the regulatory feedback loop between miR-124 and PKM1/HNF4alpha in colorectal cancer cells. Int J Mol Sci 15(3):4318-4332

3. Liu L, Chen L, Xu Y, Li R, Du X (2010) microRNA-195 promotes apoptosis and suppresses tumorigenicity of human colorectal cancer cells. Biochem Biophys Res Commun 400(2):236-240

4. Zhang H, Li Y, Huang Q, Ren X, Hu H, Sheng H, Lai M (2011) MiR-148a promotes apoptosis by targeting Bcl-2 in colorectal cancer. Cell Death Differ 18(11):1702-1710

5. Nie J, Liu L, Zheng W, Chen L, Wu X, Xu Y, Du X, Han W (2012) microRNA-365, down-regulated in colon cancer, inhibits cell cycle progression and promotes apoptosis of colon cancer cells by probably targeting Cyclin D1 and Bcl-2. Carcinogenesis 33(1):220-225

6. Gong J, Zhang JP, Li B, Zeng C, You K, Chen MX, Yuan Y, Zhuang SM (2013) MicroRNA-125b promotes apoptosis by regulating the expression of Mcl-1, Bcl-w and IL-6R. Oncogene 32(25):3071-3079

7. Karaayvaz M, Zhai H, Ju J (2013) miR-129 promotes apoptosis and enhances chemosensitivity to 5-fluorouracil in colorectal cancer. Cell Death Dis 4:e659

8. Li WH, Wu HJ, Li YX, Pan HG, Meng T, Wang X (2016) MicroRNA-143 promotes apoptosis of osteosarcoma cells by caspase-3 activation via targeting Bcl-2. Biomed Pharmacother 80:8-15

9. Funamizu N, Lacy CR, Kamada M, Yanaga K, Manome Y (2015) MicroRNA-203 induces apoptosis by upregulating Puma expression in colon and lung cancer cells. Int J Oncol 47(5):1981-1988

10. Slattery ML, Potter J, Caan B, Edwards S, Coates A, Ma KN, Berry TD (1997) Energy balance and colon cancer-beyond physical activity. Cancer Res 57(1):75-80

11. Slattery ML, Caan BJ, Benson J, Murtaugh M (2003) Energy balance and rectal cancer: an evaluation of energy intake, energy expenditure, and body mass index. Nutr Cancer 46(2):166-171

12. Slattery ML, Herrick JS, Pellatt DF, Stevens JR, Mullany LE, Wolff E, Hoffman MD, Samowitz WS, Wolff RK (2016) MicroRNA profiles in colorectal carcinomas, adenomas and normal colonic mucosa: variations in miRNA expression and disease progression. Carcinogenesis 37(3):245-261

13. Slattery ML, Mullany LE, Sakoda L, Samowitz WS, Wolff RK, Stevens JR, Herrick JS (2018) The NF-kappaB signalling pathway in colorectal cancer: associations between dysregulated gene and miRNA expression. J Cancer Res Clin Oncol 144(2):269-283

14. Slattery ML, Mullany LE, Sakoda LC, Wolff RK, Stevens JR, Samowitz WS, Herrick JS (2018) The PI3K/AKT signaling pathway: associations of miRNAs with dysregulated gene expression in colorectal cancer. Mol Carcinog 57(2):243-261

15. Slattery ML, Herrick JS, Mullany LE, Valeri N, Stevens J, Caan BJ, Samowitz W, Wolff RK (2015) An evaluation and replication of miRNAs with disease stage and colorectal cancer-specific mortality. Int J Cancer 137(2):428-438

16. Pellatt AJ, Slattery ML, Mullany LE, Wolff RK, Pellatt DF (2016) Dietary intake alters gene expression in colon tissue: possible underlying mechanism for the influence of diet on disease. Pharmacogenet Genomics 26(6):294

17. Slattery ML, Pellatt DF, Mullany LE, Wolff RK, Herrick JS (2015) Gene expression in colon cancer: a focus on tumor site and molecular phenotype. Genes Chromosom Cancer 54(9):527-541

18. Pellatt DF, Stevens JR, Wolff RK, Mullany LE, Herrick JS, Samowitz W, Slattery ML (2016) Expression profiles of miRNA subsets distinguish human colorectal carcinoma and normal colonic mucosa. Clin Transl Gastroenterol 7:e152

19. Agilent GeneSpring User Manual

20. Benjamini Y, Hochberg Y (1995) Controlling the false discovery rate: a practical and powerful approach to multiple testing. J R Stat Soc 57:289-300

21. Mullany LE, Herrick JS, Wolff RK, Slattery ML (2016) MicroRNA seed region length impact on target messenger RNA expression and survival in colorectal cancer. PLoS ONE 11(4):e0154177

22. Karolchik D, Hinrichs AS, Furey TS, Roskin KM, Sugnet CW, Haussler D, Kent WJ (2004) The UCSC table browser data retrieval tool. Nucleic Acids Res 32(Database issue):D493-D496

23. Lin Y, Zhang Q, Zhang HM, Liu W, Liu CJ, Li Q, Guo AY (2015) Transcription factor and miRNA co-regulatory network reveals shared and specific regulators in the development of B cell and T cell. Sci Rep 5:15215

24. Martinez NJ, Walhout AJ (2009) The interplay between transcription factors and microRNAs in genome-scale regulatory networks. Bioessays 31(4):435-445

25. Mangan S, Alon U (2003) Structure and function of the feed-forward loop network motif. Proc Natl Acad Sci USA 100(21):11980-11985

26. Chwieralski CE, Welte T, Buhling F (2006) Cathepsin-regulated apoptosis. Apoptosis 11(2):143-149

27. Gormley JA, Hegarty SM, O'Grady A, Stevenson MR, Burden RE, Barrett HL, Scott CJ, Johnston JA, Wilson RH, Kay EW et al (2011) The role of Cathepsin S as a marker of prognosis and predictor of chemotherapy benefit in adjuvant CRC: a pilot study. $\mathrm{Br}$ J Cancer 105(10):1487-1494

28. Wang B, Sun J, Kitamoto S, Yang M, Grubb A, Chapman HA, Kalluri R, Shi GP (2006) Cathepsin S controls angiogenesis and tumor growth via matrix-derived angiogenic factors. J Biol Chem 281(9):6020-6029

29. Nakano H, Miyazawa T, Kinoshita K, Yamada Y, Yoshida T (2010) Functional screening identifies a microRNA, miR-491 that induces apoptosis by targeting $\mathrm{Bcl}-\mathrm{X}(\mathrm{L})$ in colorectal cancer cells. Int J Cancer 127(5):1072-1080

30. Kuwano Y, Nishida K, Kajita K, Satake Y, Akaike Y, Fujita K, Kano S, Masuda K, Rokutan K (2015) Transformer 2beta and miR-204 regulate apoptosis through competitive binding to $3^{\prime}$ UTR of BCL2 mRNA. Cell Death Differ 22(5):815-825

31. Nakazawa K, Dashzeveg N, Yoshida K (2014) Tumor suppressor p53 induces miR-1915 processing to inhibit Bcl-2 in the apoptotic response to DNA damage. FEBS J 281(13):2937-2944

32. Slattery ML, Herrick JS, Stevens JR, Wolff RK, Mullany LE (2017) An assessment of database-validated microRNA target genes in normal colonic mucosa: implications for pathway analysis. Cancer Inform 16:1176935117716405

33. Altieri DC (2003) Survivin, versatile modulation of cell division and apoptosis in cancer. Oncogene 22(53):8581-8589 
34. Mita AC, Mita MM, Nawrocki ST, Giles FJ (2008) Survivin: key regulator of mitosis and apoptosis and novel target for cancer therapeutics. Clin Cancer Res 14(16):5000-5005

35. Bian K, Fan J, Zhang X, Yang XW, Zhu HY, Wang L, Sun JY, Meng YL, Cui PC, Cheng SY et al (2012) MicroRNA-203 leads to G1 phase cell cycle arrest in laryngeal carcinoma cells by directly targeting survivin. FEBS Lett 586(6):804-809

36. Chou CH, Chang NW, Shrestha S, Hsu SD, Lin YL, Lee WH, Yang CD, Hong HC, Wei TY, Tu SJ et al (2016) miRTarBase
2016: updates to the experimentally validated miRNA-target interactions database. Nucleic Acids Res 44(D1):D239-D247

37. Guo H, Ingolia NT, Weissman JS, Bartel DP (2010) Mammalian microRNAs predominantly act to decrease target mRNA levels. Nature 466(7308):835-840

38. Baek D, Villen J, Shin C, Camargo FD, Gygi SP, Bartel DP (2008) The impact of microRNAs on protein output. Nature 455(7209):64-71 